\title{
Outcomes of mid-term and long-term degradable biosynthetic meshes in single-stage open complex abdominal wall reconstruction
}

\author{
J. J. M. Claessen ${ }^{1}$ (1) A. S. Timmer ${ }^{1}$ J. J. Atema ${ }^{1}$ M. A. Boermeester ${ }^{1}$
}

Received: 6 January 2021 / Accepted: 12 April 2021 / Published online: 7 June 2021

(c) The Author(s) 2021

\begin{abstract}
Objective To assess clinical outcomes in patients that underwent open single-stage complex abdominal wall reconstruction (CAWR) with biosynthetic mesh.

Methods Retrospective observational study of two prospectively registered series of consecutive patients undergoing CAWR with either long-term degradable (LTD) Phasix ${ }^{\mathrm{TM}}$ or mid-term degradable (MTD) BIO-A ${ }^{\circledR}$ biosynthetic mesh in a single institution between June 2016 and December 2019.

Results From 169 patients with CAWR, 70 consecutive patients were identified who underwent CAWR with either LTD or MTD biosynthetic mesh. More than $85 \%$ of patients had an incisional hernia that could be classified as moderately complex to major complex due to a previous wound infection $(67 \%)$, one or more complicating comorbidities $(87.1 \%)$, one or more complicating hernia characteristics $(75.7 \%)$ or contaminated or dirty defects $(37.1 \%)$. Concomitant component separation was performed in 43 of 70 patients (61.4\%). Overall surgical site infection (SSI) rate in these CAWR patients was $45.7 \%$. Seventeen of 70 patients (24.3\%) had computed tomography (CT) - and culture-confirmed SSI in direct contact of mesh, suspicious of mesh infection. Mesh removal for persistent local infection occurred in 10\% (7 of 70) after a median of 229 days since surgery. Salvage rate of mesh after direct contact with infection was 58.8\%. All removed meshes were in the LTD group. Seven patients (10\%) had a recurrence; four patients in the LTD group (10\%) had a recurrence at a median follow-up of 35 months and three patients in the MTD group (10\%) at a median follow-up of 11 months. Three of the seven recurrences occurred in patients with SSI in persistent and direct contact with mesh.

Conclusions Comorbid patients undergoing open complex abdominal wall reconstruction are at high risk of postoperative wound complications regardless of which type of biosynthetic mesh is used. When in persistent and direct contact with infection, long-term biodegradable biosynthetic meshes may need to be removed, whereas mid-term biodegradable biosynthetic meshes can be salvaged.
\end{abstract}

Keywords Biosynthetic mesh $\cdot$ CAWR $\cdot$ mid-term degradable $\cdot$ long-term degradable $\cdot$ Bio-A $\cdot$ Phasix

\section{Introduction}

Ventral hernia development is one of the most common complications following laparotomy [1]. Where suture repair is associated with unacceptably high recurrence rates, the use

Jeroen J.M. Claessen and Allard S. Timmer shared first authorship as both have contributed equally to this manuscript.

M. A. Boermeester

m.a.boermeester@amsterdamumc.nl

1 Department of Surgery, Amsterdam Gastroenterology and Metabolism, Infection and Immunity, Amsterdam UMC, Location AMC, University of Amsterdam, Amsterdam, The Netherlands of synthetic mesh has acceptable results following abdominal wall reconstruction (AWR) [2]. Patient comorbidities and reconstructions in contaminated hernia sites are associated with postoperative wound complications [3]. The risk of surgical site infection (SSI) and (chronic) mesh infection with the possible need for mesh removal has resulted in surgeons' reluctance to use permanent synthetic mesh in high-risk settings.

Biologic mesh serves as a temporary scaffold, facilitating revascularization and remodeling of the native abdominal wall [4]. It is proposed that these meshes can withstand or reduce bacterial contamination. Multiple studies investigating the use of biologic mesh in high-risk patients and/or contaminated hernia repair found that most postoperative wound 
complications can indeed be managed conservatively, and the number of patients that develop mesh infection requiring removal is as low as $0 \%$ in non-crosslinked biologic mesh [3, 5-7]. However, reported hernia recurrence rates could be as high as $30 \%$ after 3 years [7, 8]. These results combined with the high purchase costs of biologic meshes have led to a search in alternative meshed to be used in complex AWR (CAWR).

Biosynthetic mesh, also known as biodegradable or bioabsorbable mesh, is composed of absorbable synthetic material and aims to provide the same advantages as biologic mesh, providing a more durable repair but at lower cost. Currently, three biosynthetic meshes are available on the market: BIO-A ${ }^{\circledR}$ (Gore, Flagstaff, AZ, USA), Phasix ${ }^{\mathrm{TM}}$ mesh (CR Bard Inc., Murray Hill, NJ, USA), and TIGR Matrix ${ }^{\mathrm{TM}}$ (Novus Scientific, Uppsala, Sweden). All three are fully resorbable through hydrolyzation in 7,18 and 36 months, respectively. Published data on biosynthetic meshes used in CAWR is limited [9-14], and it remains uncertain how these meshes perform when used in high-risk patients and contaminated fields or when in contact with a postoperative SSI. Whether native tissue ingrowth into the temporary scaffold provided by a biosynthetic mesh results in high-quality support to the reconstruction is also in need of more clinical data.

The aim of this study is to assess clinical outcomes in high-risk patients that underwent open single-stage CAWR with one of two types of biosynthetic mesh: a long-term degradable and a mid-term degradable biosynthetic mesh.

\section{Methods}

\section{Study design}

In this observational study, we retrospectively analyzed two prospectively registered series of consecutive patients in which a biosynthetic mesh, either a long-term degradable (LTD) (Phasix ${ }^{\mathrm{TM}}$, CR Bard Inc.) or mid-term degradable (MTD) (BIo-A ${ }^{\circledR}$, Gore) was used for single-stage open CAWR in the Amsterdam UMC, location AMC, The Netherlands, between June 2016 and December 2019. The study was approved by the Institutional Review Board, and is reported following the Strengthening the Reporting of Observational Studies in Epidemiology (STROBE) statement [15].

\section{Inclusion criteria}

Patients were eligible for inclusion if they underwent an open single-stage CAWR using biosynthetic mesh and consented to the use of their data.

\section{Data items}

Baseline demographic data included age, gender, body mass index (BMI), smoking status, diabetes mellitus (DM), cardiac disease, chronic obstructive pulmonary disease (COPD), use of medication, number of previous abdominal surgeries and abdominal wall reconstructions, presence of stomata, intestinal fistulas and infected mesh, wound classification status according to the Centers for Disease and Control and Prevention [16], preoperative botulinum toxin A (BTA) injections, hernia width, and loss of domain measured as described by Sabbagh et al. [17]. The risk of postoperative complications and hernia recurrence were classified according to the modified Ventral Hernia Working Group grading system (mVHWG) and the hernia, patient, wound (HPW) classification [18, 19]. The mVHWG classification system does not incorporate important risk factors as hernia width and loss of domain. The HPW classification includes hernia width but not loss of domain. To better describe the complexity of patients and their abdominal wall defects, we, therefore, assigned them to one of three severity classes (minor, moderate and major complex) as described by an expert consensus group in 2014 [20].

Operative data included concomitant bowel surgery, use of a component separation technique (CST; anterior CST or transverse abdominis release (TAR)), specific mesh type, mesh location, full-thickness skin reconstruction, incisional negative pressure wound therapy (iNPWT), and posterior and anterior fascial closure. Anterior component separation (ACS) was defined as an open or endoscopic release of the aponeurosis of the external oblique muscle, $1.5 \mathrm{~cm}$ lateral to the linea semilunaris. TAR was defined as separation of the transversus abdominis muscle medial of the neurovascular bundles and the linea semilunaris (following a Rives-Stoppa approach) and further dissection over the transverse fascia. Preparation over the posterior rectus sheath with mesh placement in Rives-Stoppa was not scored as a CST.

\section{Outcome variables}

Clinical outcomes analyzed were incidence of (suspected) mesh infection and subsequent need for mesh removal for persistent infection during follow-up, salvage rate of mesh, surgical site occurrence (SSO), SSI, length of hospital stay, reoperations and hernia recurrence. Suspected mesh infection was defined as computed tomography (CT)- and cultureconfirmed direct contact of mesh with SSI. Surgical site infection was divided into superficial, deep and organ space according to CDC criteria [16]. Recurrence was assessed during visits to the outpatient clinic and subsequently confirmed with CT. Furthermore, all patients were contacted by telephone and asked if they had visited any other hospital 
for diagnostics or treatment of their abdominal wall. Patients who did not perceive a recurrence, and declared absence of bulging and pain at the site of the repair, were defined as recurrence free, as a negative reply to these questions has a negative predictive value of $94 \%$ for hernia recurrence [21].

Due to the COVID-19 pandemic, we did not invite patients who reported symptoms in line with a possible recurrence for clinical assessment. For patients who deceased during the study period, follow-up extended to the last outpatient visit.

\section{Analysis}

All data items are summarized for the entire group, and in subgroups according to the specific type of biosynthetic mesh used. Numerical data are expressed as mean and standard deviation (SD) or median and range. Categorical data are summarized as count and percentage. Preoperative and operative data were compared using the unpaired $t$ test and Chi square or Fisher exact test for numerical and categorical data respectively, with a significant level $p<0.05$.

Due to differences in mesh choice based on patient and hernia characteristics over time (Fig. 1), the LTD and MTD mesh groups are two independent series of consecutive patients. In 2019, in more contaminated or major complex cases, we started to use Ovitex ${ }^{\circledR}$ reinforced tissue matrix, a hybrid biological mesh (TELA Bio, Malvern, PA, USA), and we used the MTD biosynthetic mesh BIO-A ${ }^{\circledR}$ for mild or moderately contaminated hernia repair and without risk of needing a bridging repair. Only further forward in time did we use MTD biosynthetic mesh for more complex cases. Therefore, the LTD and MTD biosynthetic mesh groups are a priori not comparable on hernia and surgery characteristics, and as such postoperative outcomes were not statistically compared between the two mesh groups. However, we did assess the cumulative recurrence rate during follow-up using Kaplan-Meier analysis (log rank). The incidence between both groups is compared at the time point at which one-third of patients from one cohort was still at risk of recurrence (censored analysis).

\section{Treatment strategy}

The tailored treatment differed between patients, but in general treatment strategy was as follows. All patients were preoperatively assessed and optimized by a multidisciplinary team. In short; patients with intestinal failure were optimized according our in-house protocol supervised by our intestinal failure team [22, 23]. Patients with a complex abdominal wall defect and identified risk factors (e.g., smoking, obesity, diabetes, malnutrition, COPD) were optimized during a prehabilitation period before surgery. As of October 2018, patients with defect widths over $10 \mathrm{~cm}$ and/or $\geq 20 \%$ loss of domain were treated with preoperative BTA injections bilateral in the lateral abdominal wall musculature.

Mesh choice depended on the complexity of the patients and the reconstruction, as well as the type of mesh available at that time period. A timeline of the developments in CAWR approach in our hospital is presented in Fig. 1. Two types of biosynthetic mesh were introduced in our center for more complex patients; since June 2016 a LTD biosynthetic mesh (Phasix ${ }^{\mathrm{TM}}$ ) and since November 2018 a MTD biosynthetic mesh $\left(\mathrm{BIO}-\mathrm{A}^{\circledR}\right)$.

In patients with clean defects, biosynthetic mesh was used as single mesh repair. In large and contaminated/dirty defects, a dual layer technique was used when the anterior

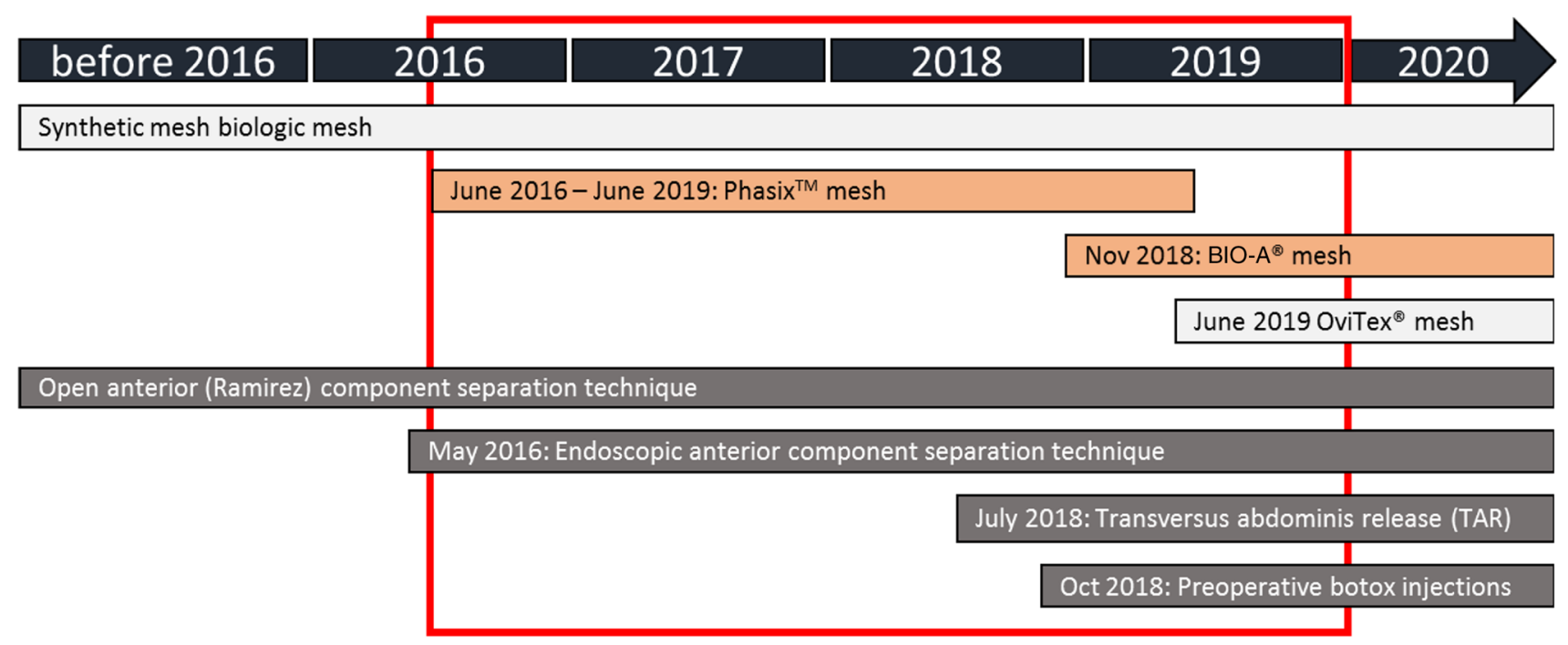

Fig. 1 Developments in CAWR in the Amsterdam UMC, location AMC 
fascia could not be closed despite a CST. This technique comprised an intra-abdominal (underlay) biologic mesh as a leverage with parachuting transfascial sutures to pull close the abdominal cavity and thereby protecting the intraabdominal viscera; a biosynthetic mesh positioned as retrorectus (sublay) reinforcement ventral to the biologic mesh creating a dual layer repair.

Superficial wound infections were treated conservatively. Deep and organ space SSIs were treated by radiological percutaneous drainage with or without antibiotics. Percutaneous drainage was also performed when a SSI was in direct contact with mesh, CT and culture confirmed, to salvage the suspected infected mesh. If the mesh location remained persistently infectious, operative placement of a negative pressure wound therapy with instillation (NPWT-I) (V.A.C. VERAFLO $^{\text {TM }}$, KCI, San Antonio, TX, USA)) was performed. When, despite this treatment, the mesh could not be salvaged during the course of several months of follow-up it was surgically removed.

\section{Surgical technique}

All operative procedures were tailored to the patient; however, all operations were performed in consistence with international guidelines and consensus [24]. The abdomen was encountered through the previous laparotomy incision, transecting and dissecting but not removing the hernia sac, followed by extensive adhesiolysis. Bioburden was reduced by resection of previously placed mesh and non-viable tissue. Enterocutaneous or enteroatmospheric fistulas were resected by segmental bowel resection, and a hand-sewn anastomosis was constructed. Up-stream diverting stomas were used infrequently when necessary. Tension-free midline closure of both fascial layers was attempted with mesh reinforcement, preventing a bridged repair whenever possible. When indicated and feasible, component separation techniques were used. If the posterior fascia could not be brought together in the midline, one side of the hernia sac was used to close the abdominal cavity. If the anterior fascia could not be closed without midline tension, or when both fascial layers could not be closed at all, a double layer mesh technique was used (see "Treatment strategy"). Biosynthetic mesh was preferably placed in the retro-rectus (sublay) plane. Subcutaneous drains were placed to the surgeon's discretion (predominantly on the mesh and when a CST was used). Prophylactic closed incision negative pressure wound therapy (NPWT; Prevena ${ }^{\mathrm{TM}}$, KCI, San Antonio, TX, USA)] for 5-7 days at $-125 \mathrm{mmHg}$ has been standard care in our practice since 2014. In the presence of significant loss of skin quality, large full-thickness skin defects and significant loss of domain, reconstruction was performed in collaboration with plastic and reconstructive surgeons.

Postoperatively all patients were instructed to wear an abdominal binder 24/7 during the first 2 weeks and subsequent for 3 months when mobilizing.

\section{Results}

\section{Participant selection and preoperative data}

A total of 169 CAWRs were performed during the study period (June 2016 and December 2019); Fig. 2 depicts the subgroups of different meshes used for CAWR. For types and combinations used during the study period (see Online Supplementary material). In 71 of the 169 reconstructions, biosynthetic mesh was used. One patient did not give informed consent. Therefore, a total of 70 patients were included; 40 patients with LTD biosynthetic mesh, and 30 with MTD biosynthetic mesh.

Baseline patient and hernia characteristics of all CAWRs performed with biosynthetic mesh in the study period are presented in Table 1. More than $85 \%$ of patients had an incisional hernia that could be classified as moderate to major complex due to a previous wound infection (67\%), one or more complicating comorbidities (87.1\%), one or more complicating hernia characteristics $(75.7 \%)$ or
Fig. 2 Meshes used of all CAWR between June 2016 and Dec 2019

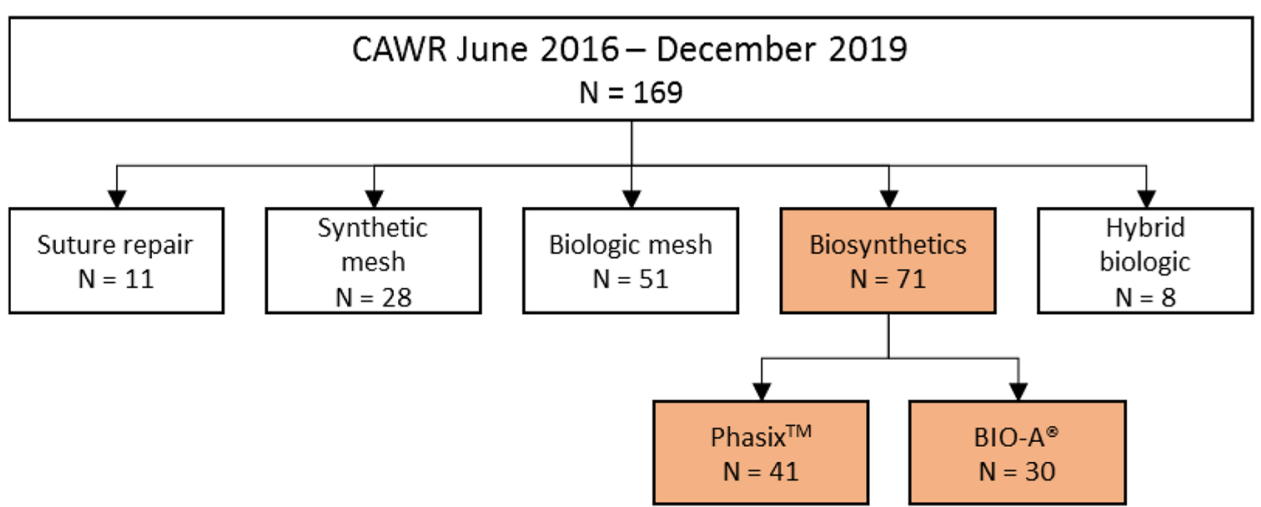


Table 1 Preoperative data

\begin{tabular}{|c|c|c|c|c|}
\hline & $\begin{array}{l}\text { Total group }(n=70) \\
\text { Mean }( \pm \mathrm{SD}), n(\%)\end{array}$ & $\begin{array}{l}\text { LTD }(n=40) \\
\text { Mean }( \pm \text { SD }), n(\%)\end{array}$ & $\begin{array}{l}\text { MTD }(n=30) \\
\text { Mean }( \pm \text { SD }), n(\%)\end{array}$ & $p$ value \\
\hline \multicolumn{5}{|l|}{ Patient demographics } \\
\hline \multicolumn{4}{|c|}{ Number of complicating comorbidities present ${ }^{\mathrm{a}}$} & 0.301 \\
\hline 0 & $9(12.9 \%)$ & $3(7.5 \%)$ & $6(20 \%)$ & \\
\hline $1-2$ & $36(51.4 \%)$ & $22(55 \%)$ & $14(46.7 \%)$ & \\
\hline$\geq 3$ & $25(35.7 \%)$ & $15(37.5 \%)$ & $10(33.3 \%)$ & \\
\hline Age (years) & $60.4( \pm 14.0)$ & $64.4( \pm 12.5)$ & $55.1( \pm 14.4)$ & 0.005 \\
\hline Male sex & $35(50 \%)$ & $20(50 \%)$ & $15(50 \%)$ & 0.999 \\
\hline BMI $\left(\mathrm{kg} / \mathrm{m}^{2}\right)$ & $29.1( \pm 5.1)$ & $29.0( \pm 4.4)$ & $29.0( \pm 5.9)$ & 0.889 \\
\hline \multicolumn{4}{|l|}{ Smoking status } & 0.563 \\
\hline Active & $13(18.6 \%)$ & $8(20 \%)$ & $5(16.7 \%)$ & \\
\hline Stopped & $34(48.6 \%)$ & $21(52.5 \%)$ & $13(43.4 \%)$ & \\
\hline Non smoker & $23(32.9 \%)$ & $11(27.5 \%)$ & $12(40.0 \%)$ & \\
\hline Diabetes mellitus & $12(17.1 \%)$ & $7(17.5 \%)$ & $5(16.7 \%)$ & 0.927 \\
\hline Cardiac disease & $14(20 \%)$ & $7(17.5 \%)$ & $7(23.2 \%)$ & 0.764 \\
\hline COPD & $11(15.7 \%)$ & $10(25 \%)$ & $1(3.3 \%)$ & 0.019 \\
\hline Anticoagulative medication & $19(27.1 \%)$ & $12(30 \%)$ & $7(23.3 \%)$ & 0.535 \\
\hline Immunosuppressive medication & $4(5.7 \%)$ & $2(5 \%)$ & $2(6.7 \%)$ & 0.999 \\
\hline Previous SSI & $47(67 \%)$ & $27(67.5 \%)$ & $20(66.7 \%)$ & 0.941 \\
\hline \multicolumn{5}{|l|}{ Hernia and wound characteristics } \\
\hline \multicolumn{4}{|c|}{ Number of complicating hernia characteristics present ${ }^{b}$} & 0.048 \\
\hline 0 & $17(24.3 \%)$ & $10(25 \%)$ & $7(23.3 \%)$ & \\
\hline $1-2$ & $34(48.6 \%)$ & $15(37.5 \%)$ & $19(63.3 \%)$ & \\
\hline$\geq 3$ & $19(27.1 \%)$ & $15(37.5 \%)$ & $4(13.4 \%)$ & \\
\hline \multicolumn{4}{|l|}{ Hernia severity class } & 0.733 \\
\hline Minor complex & $10(14.3 \%)$ & $5(12.5 \%)$ & $5(16.7 \%)$ & \\
\hline Moderate complex & $26(37.1 \%)$ & $14(35 \%)$ & $12(40 \%)$ & \\
\hline Major complex & $34(48.6 \%)$ & $21(52.5 \%)$ & $13(43.3 \%)$ & \\
\hline \multicolumn{4}{|c|}{ Modified VHWG classification, grade } & 0.190 \\
\hline 1 & $3(4.3 \%)$ & $2(5 \%)$ & $1(3.3 \%)$ & \\
\hline 2 & $41(58.6 \%)$ & $21(52.5 \%)$ & $20(66.7 \%)$ & \\
\hline 3 & $26(37.1 \%)$ & $17(42.5 \%)$ & $9(30.0 \%)$ & \\
\hline \multicolumn{4}{|l|}{ HPW classification, stage } & 0.442 \\
\hline 1 & $8(11.4 \%)$ & $3(7.5 \%)$ & $5(16.7 \%)$ & \\
\hline 2 & $35(50 \%)$ & $20(50 \%)$ & $15(50 \%)$ & \\
\hline 3 & $27(38.6)$ & $17(42.5 \%)$ & $10(33.3 \%)$ & \\
\hline Stoma present & $11(15.7 \%)$ & $9(22.5 \%)$ & $2(6.7 \%)$ & 0.072 \\
\hline Intestinal fistula(s) present & $12(17.1 \%)$ & $10(25 \%)$ & $2(6.7 \%)$ & 0.044 \\
\hline Infected mesh present & $6(8.6 \%)$ & $3(7.5 \%)$ & $3(10.0 \%)$ & 0.999 \\
\hline \multicolumn{4}{|l|}{ Previous abdominal surgeries } & 0.573 \\
\hline $0-2$ & $21(30 \%)$ & $14(35 \%)$ & $7(23.3 \%)$ & \\
\hline $3-4$ & $19(27.2 \%)$ & $10(25 \%)$ & $9(30.0 \%)$ & \\
\hline$\geq 5$ & $30(42.8 \%)$ & $16(40 \%)$ & $14(46.7 \%)$ & \\
\hline \multicolumn{4}{|l|}{ Previous hernia repairs } & 0.427 \\
\hline 0 & $33(47.1 \%)$ & $21(52 \%)$ & $12(40 \%)$ & \\
\hline 1 & $14(20 \%)$ & $6(15 \%)$ & $8(26.7 \%)$ & \\
\hline$\geq 2$ & $23(32.9 \%)$ & $13(32.5 \%)$ & $10(33.3 \%)$ & \\
\hline \multicolumn{4}{|l|}{ CDC classification } & 0.134 \\
\hline
\end{tabular}


Table 1 (continued)

\begin{tabular}{|c|c|c|c|c|}
\hline & $\begin{array}{l}\text { Total group }(n=70) \\
\text { Mean }( \pm \mathrm{SD}), n(\%)\end{array}$ & $\begin{array}{l}\text { LTD }(n=40) \\
\text { Mean }( \pm \text { SD }), n(\%)\end{array}$ & $\begin{array}{l}\text { MTD }(n=30) \\
\text { Mean }( \pm \mathrm{SD}), n(\%)\end{array}$ & $p$ value \\
\hline 1 & $44(62.9 \%)$ & $23(57.5 \%)$ & $21(70.0 \%)$ & \\
\hline 2 & $9(12.9 \%)$ & $4(10 \%)$ & $5(16.7 \%)$ & \\
\hline 3 & $10(14.3 \%)$ & $9(22.5 \%)$ & $1(3.3 \%)$ & \\
\hline 4 & $7(10 \%)$ & $4(10 \%)$ & $3(10 \%)$ & \\
\hline Preoperative botox injections & $18(25.7 \%)$ & - & $18(60.0 \%)$ & 0.001 \\
\hline Hernia width $(\mathrm{cm})$, median (range) & $10.0(0-30)$ & $10.0(0-30)$ & $10.0(0-21)$ & 0.728 \\
\hline Hernia width $\geq 10 \mathrm{~cm}$ & $30(42.9 \%)$ & $18(45 \%)$ & $12(40 \%)$ & 0.676 \\
\hline Loss of domain (\%), median (range) & $10.0(0-52)$ & $11.0(0-40)$ & $10.0(0-52)$ & 0.651 \\
\hline Loss of domain $\geq 20 \%$ & $9(12.9 \%)$ & $7(17.5 \%)$ & $2(6.7 \%)$ & 0.180 \\
\hline
\end{tabular}

${ }^{a}$ Including: active smoking, BMI $>30, C O P D$ cardiac disease, $D M$ anticoagulative medication, immunosuppressive medication, previous wound infection

${ }^{b}$ Including: presence of a stoma, intestinal fistula, infected mesh, transverse defect width $\geq 10 \mathrm{~cm}$, loss of domain $>20 \%$, previous hernia repair, concomitant bowel surgery

$p$-value in bold reflects significant level $p<0.05$

Table 2 Surgical data

\begin{tabular}{lcccc}
\hline & $\begin{array}{l}\text { Total group }(n=70) \\
n(\%)\end{array}$ & $\begin{array}{l}\text { LTD }(n=40) \\
n(\%)\end{array}$ & $\begin{array}{l}\text { MTD }(n=30) \\
n(\%)\end{array}$ & $p$ value \\
\hline Concomitant bowel surgery & $17(24.3 \%)$ & $13(32.5 \%)$ & $4(13.3 \%)$ & 0.064 \\
Component separation (CST) & & & & 0.058 \\
No CST & $27(38.6 \%)$ & $15(37.5 \%)$ & $12(40.0 \%)$ & \\
Open ACS & $25(35.7 \%)$ & $18(45 \%)$ & $7(23.3 \%)$ & \\
Endo ACS & $4(5.7 \%)$ & $3(7.5 \%)$ & $1(3.3 \%)$ & \\
Open TAR & $14(20 \%)$ & $4(10 \%)$ & $10(33.3 \%)$ & \\
Mesh type & & & & 0.076 \\
Biosynthetic only & $43(61.4 \%)$ & $21(52.5 \%)$ & $22(73.3 \%)$ & \\
Additional strattice & $18(25.7 \%)$ & $14(35 \%)$ & $4(13.3 \%)$ & \\
Additional XenMatrix & $5(7.0 \%)$ & $5(12.5 \%)$ & & - \\
Additional OviTex & $2(2.9 \%)$ & & $2(6.7 \%)$ & \\
Additional Vypro & $2(2.9 \%)$ & - & $2(6.7 \%)$ & \\
Biosynthetic mesh location & & & & $\mathbf{0 . 0 0 3}$ \\
Retro-rectus (sublay) & $52(74.3 \%)$ & $24(60 \%)$ & $28(93.3 \%)$ & \\
Intra-abdominal (underlay) & $3(4.3 \%)$ & $2(5 \%)$ & $1(3.3 \%)$ & \\
Onlay reinforcement of biologic & $9(12.9 \%)$ & $9(22.5 \%)$ & & - \\
Inlay reinforcement of biologic & $6(8.6 \%)$ & $5(12.5 \%)$ & $1(3.3 \%)$ & \\
Major full-thickness skin reconstruction & $15(21.4 \%)$ & $10(25 \%)$ & $5(16.7 \%)$ & 0.400 \\
Postoperative incisional NPWT & $62(88.6 \%)$ & $36(90 \%)$ & $26(86.7 \%)$ & 0.717 \\
Fascial closure & & & & $\mathbf{0 . 0 3 5}$ \\
Anterior + posterior fascia closed & $53(75.7 \%)$ & $28(70 \%)$ & $25(83.3 \%)$ & \\
Posterior fascia closed only & $2(2.9 \%)$ & - & $2(6.7 \%)$ & \\
Bridged repair (anterior nor posterior & $15(21.4 \%)$ & $12(30 \%)$ & $3(10 \%)$ & \\
$\quad$ fascia closed) & & & & \\
\hline
\end{tabular}

CST component separation technique, open ACS anterior component separation (Ramirez), endo ACS endoscopic anterior component separation technique, open TAR transversus abdominis release $p$-value in bold reflects significant level $p<0.05$ 
contaminated or dirty defects (37.1\%). Sixty-seven percent of patients had experienced a previous SSI prior to their indexed CAWR at our institute. Only three patients were had a $\mathrm{mVHWG}$ grade I hernias.

\section{Surgical data}

Surgical data are presented in Table 2. Concomitant bowel surgery was performed in $24.3 \%$ (17 patients). A CST was performed in $61.4 \%$ of patients (43 of 70 patients) with open ACS in $35.7 \%$ and TAR in $20 \%$ of patients. Biosynthetic mesh only was used $43(61.4 \%)$ times, whereas in 23 (32.7\%) an additional biologic mesh, and in four (5.8\%) an additional synthetic or hybrid biologic mesh was used.

\section{Differences in preoperative and operative data between LTD and MTD biosynthetic mesh}

Presence of COPD and intestinal fistula(s) was much higher in the LTD group $(25.0 \%$ vs $3.3 \% p<0.019$ and $25.0 \%$ vs $6.7 \% p<0.044$, respectively), associated with a change in mesh indications over time in our practice. Injection of BTA was performed in $60.0 \%$ of patients in the MTD group, and in only $2.4 \%$ of the LTD group, associated with the change in practice over time $(p<0.001)$. Biosynthetic mesh positioning differed significantly between groups $(p<0.003)$, relating to differences in hernia complexity among the two groups. Biosynthetic mesh was mostly placed in retromuscular (sublay) position in $93.3 \%$ of the MTD group vs. $60 \%$ of LTD group. Biosynthetic mesh was used as onlay reinforcement after primary fascial closure in 9 patients $(22.5 \%)$ of the LTD group versus none in the MTD group.

\section{Clinical outcomes}

Clinical outcomes are presented in Table 3. Overall SSI rate in these CAWR patients was $45.7 \%$. Seventeen of 70 patients (24.3\%) had a SSI in direct contact with mesh on CT, suspicious of mesh infection; of which 10 of 40 patients (25.0\%) in the LTD group and 7 of 30 patients (23.3\%) in the MTD group. Ten of those 17 patients with SSI in direct contact with mesh had the biosynthetic mesh in the retro-rectus position (4 of 10 in the LTD group and 6 of 7 in the MTD group). In 7 of 70 patients (10\%) and 7 of 17 meshes (41.2\%) with persistent local infection, the mesh had to be removed after a median of 229 days since surgery. All 7 mesh removals occurred in the LTD group and none in the MTD group. The mesh salvage rate after in direct contact with SSI was $58.8 \%$ (30\% in the LTD group and 100\% in the MTD group).

At a median follow-up of 20 months (range 3-46), 7 of 70 patients (10\%) had a CT-confirmed recurrent hernia. Median follow-up was 35 months for the LTD group and 11 months for the MTD group. Four of 40 patients (10\%) had a recurrence in the LTD group and three of 30 patients $(10 \%)$ in the MTD group. Two additional patients in the MTD reported symptoms that indicated a possible recurrence. Three of the seven recurrences occurred in patients with SSI in persistent and direct contact with mesh (two patients in the LTD group and one in the MTD group; in the two LTD patients, the mesh needed to be removed).

The cumulative recurrence rate at 13 months postoperative (time at which 1/3 of MTD cohort was still at risk for recurrence) was not statistically different between both groups ( $p=0.792$; see Online supplementary material).

\section{Discussion}

The importance of mesh reinforcement to decrease recurrence rates has been widely acknowledged. However, which mesh to use in which type of patients remains an ongoing subject of discussion [25]. Because patient and hernia characteristics greatly affect clinical outcome and the risk of postoperative wound complications, just as meshes can do, it is difficult to assess the influence of a specific mesh.

Mesh infection with the subsequent need of mesh removal is a feared complication of CAWR. It is, therefore, important to know how the mesh behaves in postoperative local infections; can it be salvaged or does it need to be removed?

Present study evaluates clinical outcomes of two independent series of 70 consecutive patients in total, with moderate to highly complex incisional hernias who underwent single-stage open CAWR using LTD $(n=40)$ or MTD $(n=30)$ biosynthetic mesh. With a postoperative SSI rate of $45.7 \%$ and mesh in direct contact with SSI, suspicious of mesh infection, occurring in $24.3 \%$ of patients, present study shows that when using biosynthetic meshes in complex patients, short-term outcomes remain challenging. Mesh was removed in $10 \%$ of patients because of persistent local infection, giving a salvage rate of nearly $60 \%$. The hernia recurrence rate was $10 \%$ at a median follow-up of 20 months.

Comparison with other studies with respect to mesh infection is difficult as studies use various definitions for mesh infections. We were very strict in our evaluation of mesh behavior in infected environment and defined mesh infection as CT- and culture-confirmed direct contact of mesh with SSI. With comparable incidence in postoperative mesh infection in both biosynthetic mesh groups, it is interesting to see that the need for mesh removal for persistent infection occurred in 7 of 70 patients $(10 \%)$, all in the LTD group (seven out of ten infected meshes). It seems that when LTD mesh comes into contact with an infection, few meshes can withstand this and can be salvaged.

The higher rate of SSI could be explained by the percentage of patients (67\%) with previously experienced SSI, which compared to most studies was at least twice as high 
Table 3 Clinical outcomes

\begin{tabular}{|c|c|c|c|}
\hline & $\begin{array}{l}\text { Total group }(n=70) \\
n(\%)\end{array}$ & $\begin{array}{l}\operatorname{LTD}(n=40) \\
n(\%)\end{array}$ & $\begin{array}{l}\text { MTD }(n=30) \\
n(\%)\end{array}$ \\
\hline Suspected mesh infection because of direct contact with SSI on $\mathrm{CT}^{\mathrm{a}}$ & $17(24.3 \%)$ & $10(25 \%)$ & $7(23.3 \%)$ \\
\hline After CDC 1 or 2 repair & $12(17.1 \%)$ & $5(12.5 \%)$ & $7(23.3 \%)$ \\
\hline After CDC 3 or 4 repair & $5(7.1 \%)$ & $5(12.5 \%)$ & - \\
\hline Suspected mesh infection, mesh position & $17(24.3 \%)$ & $10(25 \%)$ & $7(23.3 \%)$ \\
\hline Retro-rectus (sublay) & $10(14.2 \%)$ & $4(10 \%)$ & $6(20 \%)$ \\
\hline Intra-abdominal (underlay) & $2(2.9 \%)$ & $2(5 \%)$ & - \\
\hline Onlay reinforcement of biologic & $2(2.9 \%)$ & $2(5 \%)$ & - \\
\hline Inlay reinforcement of biologic & $3(4.3 \%)$ & $2(5 \%)$ & $1(3.3 \%)$ \\
\hline Removal of mesh & $7(10 \%)$ & $7(17.5 \%)$ & - \\
\hline After CDC 1 or 2 repair & $4(5.7 \%)$ & $4(10 \%)$ & \\
\hline After CDC 3 or 4 repair & $3(4.3 \%)$ & $3(7.5 \%)$ & \\
\hline Removal of mesh according to position & $7(10 \%)$ & $7(17.5 \%)$ & - \\
\hline Retro-rectus (sublay) & $3(4.3 \%)$ & $3(7.5 \%)$ & \\
\hline Intra-abdominal (underlay) & $2(2.9 \%)$ & $2(5 \%)$ & \\
\hline Onlay reinforcement of biologic & $1(1.4 \%)$ & $1(2.3 \%)$ & \\
\hline Inlay reinforcement of biologic & $1(1.4 \%)$ & $1(2.3 \%)$ & \\
\hline Days between surgery and mesh removal (median, range) & $229(77-895)$ & $229(77-895)$ & - \\
\hline Salvage rate of suspected infected meh & $10 / 17(58.8 \%)$ & $3 / 10(30 \%)$ & $7 / 7(100 \%)$ \\
\hline \multicolumn{4}{|l|}{ SSO } \\
\hline No SSO & $31(44.3 \%)$ & $14(35 \%)$ & $17(56.7 \%)$ \\
\hline SSI only & $25(35.7 \%)$ & $14(35 \%)$ & $11(36.7 \%)$ \\
\hline Wound dehiscence only & $3(4.3 \%)$ & $3(7.5 \%)$ & - \\
\hline Seroma/hematoma only & $4(5.7 \%)$ & $2(5 \%)$ & $2(6.7 \%)$ \\
\hline Combination of different SSOs & $7(10 \%)$ & $7(17 \%)$ & - \\
\hline \multicolumn{4}{|l|}{ SSI (according to CDC) } \\
\hline No SSI & $38(54.3 \%)$ & $19(47.5 \%)$ & $19(63.3 \%)$ \\
\hline Superficial SSI & $16(22.9 \%)$ & $11(27.5 \%)$ & $5(16.7 \%)$ \\
\hline Deep SSI & $2(2.9 \%)$ & $1(2.3 \%)$ & $1(3.3 \%)$ \\
\hline Organ space SSI & $9(12.8 \%)$ & $4(10 \%)$ & $5(16.7 \%)$ \\
\hline Combination of SSIs & $5(7.1 \%)$ & $5(12.5 \%)$ & - \\
\hline \multicolumn{4}{|l|}{ SSI according to mesh use } \\
\hline Biosynthetic use only & $19 / 43(44.2 \%)$ & $11 / 21(52.4 \%)$ & $8 / 22(36.4 \%)$ \\
\hline Additional other mesh & $13 / 27(48.1 \%)$ & $10 / 19(52.6 \%)$ & $3 / 8(37.5 \%)$ \\
\hline \multicolumn{4}{|l|}{ Non-mesh-related reoperations } \\
\hline Surgical treatment of $\mathrm{SSO}^{\mathrm{b}}$ & $9(12.9 \%)$ & $6(15 \%)$ & $3(10.0 \%)$ \\
\hline Anastomotic leakage & $1(1.4 \%)$ & - & $1(3.3 \%)$ \\
\hline Bowel perforation & $1(1.4 \%)$ & $1(2.5 \%)$ & - \\
\hline Preexisting diaphragm paralysis & $1(1.4 \%)$ & $1(2.5 \%)$ & - \\
\hline Recurrent hernia repair & $2(2.9 \%)$ & $2(5 \%)$ & - \\
\hline \multicolumn{4}{|l|}{ Hospital stay (days) } \\
\hline Median, range & $7(4-72)$ & $9(4-72)$ & $6(5-40)$ \\
\hline \multicolumn{4}{|l|}{ Follow-up (months) } \\
\hline Median, range & $20(3-46)$ & $35(13-46)$ & $11(3-17)$ \\
\hline Hernia recurrence & $7(10 \%)$ & $4(10 \%)$ & $3(10 \%)$ \\
\hline Suspected mesh infection ${ }^{\mathrm{a}}$ prior to recurrence & 3 & 2 & 1 \\
\hline Mesh removal prior to recurrence & 2 & 2 & - \\
\hline
\end{tabular}

${ }^{\text {a }}$ Suspected mesh infection defined as biosynthetic mesh in direct contact with SSI on CT

${ }^{\mathrm{b}}$ Including surgical treatment of deep SSI $(n=4)$, V.A.C. VeraFlo ${ }^{\mathrm{TM}}$ treatment of deep SSI $(n=2)$, evacuation of hematoma $(n=1)$, excision of infected mitek anchors/osteitis $(n=1)$, recurrent enterocutaneous fistula $(n=1)$ 
(if reported at all) $[9,10,12,14]$. This line of reasoning was also confirmed by the fact that we found that suspected mesh infection also occurred in initially non-contaminated setting. The rate of SSIs in the present study is higher in comparison with other studies using just Phasix ${ }^{\mathrm{TM}}$ [9-11, 14, 26-29] or just BIO-A ${ }^{\circledR}[12,30-32]$, in which the SSI rates ranged $0-17 \%$ and $6.2-18.3 \%$, respectively. Sahoo et al. compare different biosynthetic meshes (i.e., Phasix ${ }^{\mathrm{TM}}, \mathrm{BIO}-\mathrm{A}^{\circledR}$, and Tiger $^{\mathrm{TM}}$ ) with synthetic mesh, in clean-contaminated and contaminated wounds and report a SSI rate of 22.4\%. [33] In the biosynthetic group, no mesh infection is reported and only one mesh removal (1.7\%). Another retrospective study with multiple biosynthetic meshes reports $32.7 \%$ SSIs; mesh removal in 3.6\% [34]. Another factor affecting SSI rate is the use of open ACS, which is with $35.7 \%$ considerably higher than the above-mentioned studies of biosynthetic mesh use (range 0-20\%). Open component separation (ACS) with its larger wound beds is known to be highly associated with wound complications [35]. Figure 1 shows that endo-CST but in particular TAR was more frequently used after 2018. In addition, the introduction of preoperative botulinum toxin A in 2018 and its liberal use in our institute was able to prevent the need of CST to reach medial fascial closure. Second, for large defects, the simultaneous introduction of a hybrid biological mesh (Ovitex ${ }^{\circledR}$ reinforced tissue matrix) in our practice caused a shift from extended open anterior CST to prevent bridging repair to use of intra-abdominal underlay hybrid reinforced mesh. These two more recent advances in our practice of CAWR simplified the procedure and reduced the surgical wound surface needed for the repair. Furthermore, our vigorously complication registration and culture sampling could account for our high rate of SSIs in which we register even the smallest wound complication. We also investigated if the use of an additional biologic mesh could be an explanation since the use of biologic mesh is reported with high rates of SSI [36]. In the present series, however, SSI rate was comparable for the use of a single biosynthetic mesh or a biosynthetic combined with biologic mesh.

The recurrence rate was $10 \%$ with a median follow-up of 20 months. In the MTD group, two patients reported symptoms indicating a recurrence. Due to COVID-19, we did not invite these patients to the outpatient department and could not confirm a recurrence. The reported recurrence rate for MTD is, therefore, possibly underestimated, and may be $16.7 \%$ (5 of 30) instead of $10 \%$ (3 of 30). Recurrence rates were comparable with other studies (ranging from 0 to $17 \%$ ), although not all previously published studies used CT for assessing recurrence so the true numbers could be higher than currently published [9-13, 28-33]. For the LTD biosynthetic mesh group, the median follow-up of 35 months is one of the longest in the literature. For the MTD biosynthetic mesh group, the median follow-up of 11 months in present series is, in our opinion, too short to draw any long-term conclusions on recurrences. It is of note though that the MTD mesh is completely absorbed within 7 months [12], but recurrence rates may still increase as time passes. Three of the seven recurrences occurred in patients with SSI in persistent and direct contact with mesh as shown by CT (2 patients in the LTD group and 1 in the MTD group; in the 2 LTD patients the mesh needed to be removed). It seems that LTD biosynthetic mesh can provide an adequate durable repair, but when it comes into direct contact with a local postoperative infection, the mesh needs to be removed more often than it can be salvaged. Whether or not MTD biosynthetic mesh provides a durable repair needs to be awaited due to the relatively short follow-up period, but our results show that this mesh can be salvaged in direct presence of an infection.

\section{Strengths and limitations}

Although performing a retrospective analysis, which induces possible selection bias, we included all consecutive and prospectively registered patients treated with biosynthetic mesh without any exclusion criteria. Therefore, the results of our study reflect actual clinical outcomes of the use of these biosynthetic meshes in daily surgical practice of a CAWR tertiary referral center. Where more data are needed assessing long-term outcomes of biosynthetic mesh, the LTD cohort in our study has a median follow-up of 34 months, which is relatively long to earlier studies.

The biggest limitation of the present study is that data from both groups are not suitable for direct statistical comparison and should, therefore, be regarded as two independent series of consecutive patients.

Due to the heterogeneous nature of the referred CAWR population, a combination of meshes and techniques was sometimes needed for the CAWR. Exclusion of these patients would not reflect daily practice, and splitting the cohort would result in small and fragmented subgroups making it hard to draw any conclusions.

\section{Conclusion}

Comorbid patients undergoing open complex abdominal wall repair are at high risk of postoperative wound complications regardless of which type of biosynthetic mesh is used. When in persistent and direct contact with infection, LTD biosynthetic meshes may need to be removed, whereas MTD biosynthetic meshes can be salvaged. Present study was not set-up as comparative in nature or to determine which type of biosynthetic mesh should be used in which type of 
patient, but merely aims to show clinical outcomes of their use in daily practice. It also shows that patient factors are extremely important and that highly complex patients, and not just level of contamination, can equally affect outcomes.

Supplementary Information The online version contains supplementary material available at https://doi.org/10.1007/s10029-021-02415-7.

Funding No funding.

\section{Declarations}

Conflict of interest Jeroen J.M. Claessen, Allard S. Timmer and Jasper J. Atema declare no conflict of interest. Marja A. Boermeester reported receiving institutional grants from $\mathrm{J} \& \mathrm{~J} /$ Ethicon, $\mathrm{KCI} / 3 \mathrm{M}, \mathrm{BD}$ Bard and New Compliance; and being a speaker and/or instructor for KCI/3 M, J\&J/Ethicon, Allergan/LifeCell, Bard, Gore, GD Medical, Medtronic, and Smith \& Nephew. No other disclosures were reported.

Human and animal rights No rights were violated.

Informed consent All patients provided oral and written consent.

Open Access This article is licensed under a Creative Commons Attribution 4.0 International License, which permits use, sharing, adaptation, distribution and reproduction in any medium or format, as long as you give appropriate credit to the original author(s) and the source, provide a link to the Creative Commons licence, and indicate if changes were made. The images or other third party material in this article are included in the article's Creative Commons licence, unless indicated otherwise in a credit line to the material. If material is not included in the article's Creative Commons licence and your intended use is not permitted by statutory regulation or exceeds the permitted use, you will need to obtain permission directly from the copyright holder. To view a copy of this licence, visit http://creativecommons.org/licenses/by/4.0/.

\section{References}

1. Poulose BK, Shelton J, Phillips S, Moore D, Nealon W, Penson D et al (2012) Epidemiology and cost of ventral hernia repair: making the case for hernia research. Hernia 16(2):179-183

2. Burger JW, Luijendijk RW, Hop WC, Halm JA, Verdaasdonk EG, Jeekel J (2004) Long-term follow-up of a randomized controlled trial of suture versus mesh repair of incisional hernia. Ann Surg. 240(4):578-583 (discussion 83-5)

3. Huntington CR, Cox TC, Blair LJ, Schell S, Randolph D, Prasad $T$ et al (2016) Biologic mesh in ventral hernia repair: outcomes, recurrence, and charge analysis. Surgery 160(6):1517-1527

4. Nahabedian MY, Sosin M, Bhanot P (2018) A current review of biologic meshes in abdominal wall reconstruction. Plast Reconstr Surg 142(3 Suppl):74S-81S

5. Atema JJ, Furnee EJ, Maeda Y, Warusavitarne J, Tanis PJ, Bemelman WA et al (2017) Major complex abdominal wall repair in contaminated fields with use of a non-cross-linked biologic mesh: a dual-institutional experience. World J Surg 41(8):1993-1999

6. Itani KM, Rosen M, Vargo D, Awad SS, Denoto G 3rd, Butler CE et al (2012) Prospective study of single-stage repair of contaminated hernias using a biologic porcine tissue matrix: the RICH study. Surgery 152(3):498-505

7. Rosen MJ, Krpata DM, Ermlich B, Blatnik JA (2013) A 5-year clinical experience with single-staged repairs of infected and contaminated abdominal wall defects utilizing biologic mesh. Ann Surg 257(6):991-996

8. de Vries FEE, Hodgkinson JD, Claessen JJM, van Ruler O, Leo CA, Maeda Y et al (2020) Long-term outcomes after contaminated complex abdominal wall reconstruction. Hernia 24(3):459-468

9. Levy AS, Bernstein JL, Premaratne ID, Rohde CH, Otterburn DM, Morrison KA et al (2020) Poly-4-hydroxybutyrate (Phasix) mesh onlay in complex abdominal wall repair. Surg Endosc 8:1

10. Messa CAT, Kozak G, Broach RB, Fischer JP (2019) When the mesh goes away: an analysis of poly-4-hydroxybutyrate mesh for complex hernia repair. Plast Reconstr Surg Glob Open 7(11):e2576

11. Rognoni C, Cuccurullo D, Borsoi L, Bonavina L, Asti E, Crovella F et al (2020) Clinical outcomes and quality of life associated with the use of a biosynthetic mesh for complex ventral hernia repair: analysis of the "Italian Hernia Club" registry. Sci Rep 10(1): 10706

12. Rosen MJ, Bauer JJ, Harmaty M, Carbonell AM, Cobb WS, Matthews B et al (2017) Multicenter, prospective, longitudinal study of the recurrence, surgical site infection, and quality of life after contaminated ventral hernia repair using biosynthetic absorbable mesh: the COBRA study. Ann Surg 265(1):205-211

13. Roth JS, Anthone GJ, Selzer DJ, Poulose BK, Bittner JG, Hope WW et al (2018) Prospective evaluation of poly-4-hydroxybutyrate mesh in CDC class I/high-risk ventral and incisional hernia repair: 18-month follow-up. Surg Endosc 32(4):1929-1936

14. van Rooijen MM, Jairam AP, Tollens T, Jorgensen LN, de Vries Reilingh TS, Piessen G et al (2020) Outcomes of a new slowly resorbable biosynthetic mesh (Phasix) in potentially contaminated incisional hernias: a prospective, multi-center, single-arm trial. Int J Surg 83:31-36

15. von Elm E, Altman DG, Egger M, Pocock SJ, Gotzsche PC, Vandenbroucke JP et al (2008) The strengthening the reporting of observational studies in epidemiology (STROBE) statement: guidelines for reporting observational studies. J Clin Epidemiol 61(4):344-349

16. Mangram AJ, Horan TC, Pearson ML, Silver LC, Jarvis WR (1999) Guideline for prevention of surgical site infection, 1999 centers for disease control and prevention (CDC) hospital infection control practices advisory committee. Am J Infect Control 27(2):97-132 (quiz 3-4; discussion 96)

17. Sabbagh C, Dumont F, Robert B, Badaoui R, Verhaeghe P, Regimbeau JM (2011) Peritoneal volume is predictive of tension-free fascia closure of large incisional hernias with loss of domain: a prospective study. Hernia 15(5):559-565

18. Kanters AE, Krpata DM, Blatnik JA, Novitsky YM, Rosen MJ (2012) Modified hernia grading scale to stratify surgical site occurrence after open ventral hernia repairs. J Am Coll Surg 215(6):787-793

19. Petro CC, O'Rourke CP, Posielski NM, Criss CN, Raigani S, Prabhu AS et al (2016) Designing a ventral hernia staging system. Hernia 20(1):111-117

20. Slater NJ, Montgomery A, Berrevoet F, Carbonell AM, Chang A, Franklin M et al (2014) Criteria for definition of a complex abdominal wall hernia. Hernia 18(1):7-17

21. Sneiders D, Jairam AP, de Smet GHJ, Dawson I, van Eeghem LHA, Vrijland WW et al (2020) incisional hernia cannot be diagnosed by a patient-reported diagnostic questionnaire. J Surg Res 245:656-662

22. Atema JJ, Mirck B, Van Arum I, Ten Dam SM, Serlie MJ, Boermeester MA (2016) Outcome of acute intestinal failure. Br J Surg 103(6):701-708

23. de Vries FEE, Claessen JJM, van Hasselt-Gooijer EMS, van Ruler O, Jonkers C, Kuin W et al (2020) Bridging-to-surgery in patients with type 2 intestinal failure. J Gastrointest Surg. 22:1-1 
24. Group EF, Vaizey CJ, Maeda Y, Barbosa E, Bozzetti F, Calvo J et al (2016) European Society of Coloproctology consensus on the surgical management of intestinal failure in adults. Colorectal Dis 18(6):535-548

25. Montgomery A (2013) The battle between biological and synthetic meshes in ventral hernia repair. Hernia 17(1):3-11

26. Buell JF, Sigmon D, Ducoin C, Shapiro M, Teja N, Wynter E et al (2017) Initial experience with biologic polymer scaffold (poly4-hydroxybuturate) in complex abdominal wall reconstruction. Ann Surg 266(1):185-188

27. Bueno-Lledo J, Ceno M, Perez-Alonso C, Martinez-Hoed J, Torregrosa-Gallud A, Pous-Serrano S (2020) Biosynthetic resorbable prosthesis is useful in single-stage management of chronic mesh infection after abdominal wall hernia repair. World J Surg 45(2):443-450

28. Pakula A, Skinner R (2020) Outcomes of open complex ventral hernia repairs with retromuscular placement of poly-4-hydroxybutyrate bioabsorbable mesh. Surg Innov 27(1):32-37

29. Plymale MA, Davenport DL, Dugan A, Zachem A, Roth JS (2018) Ventral hernia repair with poly-4-hydroxybutyrate mesh. Surg Endosc 32(4): 1689-1694

30. Cho JE, Helm MC, Helm JH, Mier N, Kastenmeier AS, Gould JC et al (2019) Retro-rectus placement of bio-absorbable mesh improves patient outcomes. Surg Endosc 33(8):2629-2634

31. Garcia-Urena MA, Lopez-Monclus J, Cuccurullo D, Blazquez Hernando LA, Garcia-Pastor P, Reggio S et al (2019) Abdominal wall reconstruction utilizing the combination of absorbable and permanent mesh in a retromuscular position: a multicenter prospective study. World J Surg 43(1):149-158

32. Munoz-Rodriguez JM, Lopez-Monclus J, San Miguel Mendez C, Perez-Flecha Gonzalez M, Robin-Valle de Lersundi A, Blazquez Hernando LA et al (2020) Outcomes of abdominal wall reconstruction in patients with the combination of complex midline and lateral incisional hernias. Surgery 168(3):532-542
33. Sahoo S, Haskins IN, Huang LC, Krpata DM, Derwin KA, Poulose BK et al (2017) Early wound morbidity after open ventral hernia repair with biosynthetic or polypropylene mesh. J Am Coll Surg 225(4):472-80 e1

34. Warren J, Desai SS, Boswell ND, Hancock BH, Abbad H, Ewing JA et al (2020) Safety and efficacy of synthetic mesh for ventral hernia repair in a contaminated field. J Am Coll Surg 230(4):405-413

35. Reilingh TSD, van Goor H, Charbon JA, Rosman C, Hesselink EJ, van der Wilt GJ et al (2007) Repair of giant midline abdominal wall hernias: "components separation technique" versus prosthetic repair-interim analysis of a randomized controlled trial. World J Surg 31(4):756-763

36. Atema JJ, de Vries FEE, Boermeester MA (2016) Systematic review and meta-analysis of the repair of potentially contaminated and contaminated abdominal wall defects. Am J Surg 212(5):982

Publisher's Note Springer Nature remains neutral with regard to jurisdictional claims in published maps and institutional affiliations. 